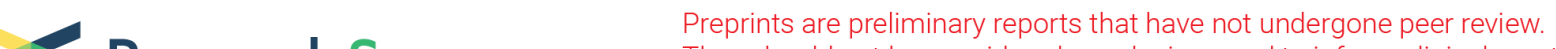 $\begin{array}{ll}\text { Research Square } & \text { They should not be considered conclusive, used to inform clinical practice, } \\ \text { or referenced by the media as validated information. }\end{array}$
}

\section{Method of Computing Direction-dependent Margins for the Development of Consensus Contouring Guidelines}

\section{Liam S. P. Lawrence}

University of Toronto https://orcid.org/0000-0003-2070-8308

\section{Lee Chin}

Sunnybrook Health Sciences Centre

Rachel W. Chan

Sunnybrook Research Institute

Timothy K. Nguyen

London Health Sciences Centre

\section{Arjun Sahgal}

Sunnybrook Health Sciences Centre

\section{Chia-Lin Tseng}

Sunnybrook Health Sciences Centre

Angus Z. Lau ( $\nabla$ angus.lau@sri.utoronto.ca )

Sunnybrook Research Institute

\section{Research Article}

Keywords: Consensus contouring, clinical target volume margins, interobserver variability, stereotactic body radiotherapy

Posted Date: February 25th, 2021

DOI: https://doi.org/10.21203/rs.3.rs-249434/v1

License: (a) (1) This work is licensed under a Creative Commons Attribution 4.0 International License. Read Full License

Version of Record: A version of this preprint was published at Radiation Oncology on April 13th, 2021. See the published version at https://doi.org/10.1186/s13014-021-01799-1. 


\section{Title page}

2 Title: Method of computing direction-dependent margins for the development of consensus

3 contouring guidelines

4

5 Authors and affiliations: Liam S. P. Lawrence, MASc, ${ }^{a}$ Lee Chin, PhD, MCCPM, ${ }^{\text {bc }}$ Rachel W. Chan,

6 PhD, ${ }^{d}$ Timothy K. Nguyen, MD, ${ }^{e}$ Arjun Sahgal, MD, ${ }^{c}$ Chia-Lin Tseng, MD, ${ }^{c}$ Angus Z. Lau, PhD ${ }^{\text {ad }}$

8 a) Department of Medical Biophysics, University of Toronto, Toronto, Ontario, Canada

9 b) Department of Medical Physics, Odette Cancer Centre, Toronto, Ontario, Canada

10 c) Department of Radiation Oncology, Sunnybrook Health Sciences Centre, University of

11 Toronto, Toronto, Ontario, Canada

12 d) Department of Physical Sciences, Sunnybrook Research Institute, Toronto, Ontario, Canada

13 e) Department of Radiation Oncology, London Health Sciences Centre, Western University, 14 London, Ontario, Canada

16 Corresponding author: Angus Z. Lau

17 Telephone number: $416-480-6100$

18 Mailing address: M7-601, 2075 Bayview Avenue, Toronto, ON Canada M4N 3M5

19 Email: angus.lau@sri.utoronto.ca 


\section{Abstract}

21 Background: Clinical target volume (CTV) contouring guidelines are frequently developed

22 through studies in which experts contour the CTV for a representative set of cases for a given

23 treatment site and the consensus CTVs are analyzed to generate margin recommendations.

24 Measures of interobserver variability are used to quantify agreement between experts. In cases

25 where an isotropic margin is not appropriate, however, there is no standard method to compute

26 margins in specified directions that represent possible routes of tumor spread. Moreover,

27 interobserver variability metrics are often measures of volume overlap that do not account for

28 the dependence of disagreement on direction. To aid in the development of consensus

29 contouring guidelines, this study demonstrates a novel method of quantifying CTV margins and

30 interobserver variability in clinician-specified directions.

32 Methods: The proposed algorithm was applied to 11 cases of non-spine bone metastases to

33 compute the consensus CTV margin in each direction of intraosseous and extraosseous disease.

34 The median over all cases for each route of spread yielded the recommended margins. The

35 disagreement between experts on the CTV margin was quantified by computing the median of 36 the coefficients of variation for intraosseous and extraosseous margins.

38 Results: The recommended intraosseous and extraosseous margins were $7.0 \mathrm{~mm}$ and $8.0 \mathrm{~mm}$,

39 respectively. The median coefficient of variation quantifying the margin disagreement between 40 experts was 0.59 and 0.48 for intraosseous and extraosseous disease. 
42 Conclusions: The proposed algorithm permits the generation of margin recommendations in

43 relation to adjacent anatomy and quantifies interobserver variability in specified directions. This

44 method can be applied to future consensus CTV contouring studies.

\section{Keywords}

46 Consensus contouring, clinical target volume margins, interobserver variability, stereotactic body

47 radiotherapy

\section{List of Abbreviations}

$49 \mathrm{CT}=$ computed tomography

$50 \quad$ CTV = clinical target volume

51 GTV = gross tumor volume

$52 \mathrm{MRI}=$ magnetic resonance imaging

53 SBRT = stereotactic body radiotherapy

54 STAPLE = simultaneous truth and performance level estimation

57 Conformal radiotherapy techniques, including stereotactic radiosurgery and stereotactic body

58 radiotherapy (SBRT), are increasingly being delivered for most malignancies. Consistent and

59 accurate delineation of the clinical target volume (CTV) is essential for ensuring treatment

60 efficacy and meaningful interpretation of dosimetric and clinical outcomes in clinical trials. The 
61 CTV is defined as an additional margin to the gross tumor volume (GTV), the demonstrable extent

62 of the tumor, to encompass microscopic disease. Ideally, CTV margin recommendations are

63 informed by pathological evaluation of microscopic tumor extension (1-3) and examination of

64 patterns of disease recurrence. In the absence of such data, expert consensus can provide

65 guidance. Over the last decade, a number of consensus contouring studies to formulate

66 guidelines have emerged for various disease sites (4-12).

67

68 In these studies, experts are typically provided with patient images and asked to contour the CTV

69 given a reference GTV. Measures of inter-observer variability are used to determine the

70 agreement between experts (13). A consensus CTV is often created from a collection of expert

71 contours by applying the simultaneous truth and performance level estimation (STAPLE)

72 algorithm (14). Consensus contouring recommendations typically include CTV margins based, in

73 part, on the STAPLE consensus CTV. In clinical contouring practice, the GTV would be expanded

74 isotropically or anisotropically along the Cartesian axes using these margins.

76 However, tumor spread can vary in extent in arbitrary directions because of anatomic barriers,

77 anisotropic tissue structure, or preferential migration within the tissue of origin (15). Future

78 treatment planning systems should implement expansion in arbitrary directions using anisotropic

79 margins for better conformation to the disease volume. For this purpose, a method of analyzing

80 expert contours to generate margin recommendations in specified directions that represent

81 potential routes of tumor spread is needed. Such analysis would also benefit from a metric for

82 quantifying interobserver variability in specified directions to assess disagreement on the

\section{4 of 16}


83 appropriate margin. Common measures of interobserver variability like the Dice coefficient and

84 the kappa statistic (17) are based on volume overlap and therefore do not capture the

85 dependence of disagreement on direction. Existing algorithms that could be applied for

86 computation of anisotropic margins and contour variability rely on approximately spherical

87 volumes $(18,19)$, which may not be a valid assumption, or do not make use of specified directions $88(20-22)$.

90 This study proposes a novel method to compute consensus CTV margins and margin variability in

91 directions chosen based on clinical assessment of potential routes of spread and anatomical

92 barriers. For generating clinical contouring recommendations, the consensus CTV margin is

93 computed for specified routes of spread in multiple cases, then a margin recommendation is

94 made based on a summary statistic across all cases. For demonstration, the method is applied to

9511 cases of non-spine bone metastases, where margins are expected to be anisotropic, to 96 determine separate intraosseous and extraosseous margins.

\section{Methods and Materials}

\section{$98 \quad$ Metrics}

99 The upcoming metric definitions rely on a quantity called the directional margin $M(\boldsymbol{d})$, where

$100 \boldsymbol{d}$ is any clinically relevant direction in 3D. The directional margin is computed from a GTV and a

101 CTV in two steps. First, a set of $N$ expansion vectors $\left\{\boldsymbol{v}_{i}\right\}_{i=1}^{N}$ are identified: these vectors are 102 oriented parallel to $\boldsymbol{d}$ and extend from the GTV surface to the CTV surface without intersecting

\section{5 of 16}


103 the interior of the GTV. Second, the directional margin is defined as the median of the lengths of

104 the expansion vectors: $M(\boldsymbol{d})=\operatorname{MEDIAN}\left(\left\{\left|\boldsymbol{v}_{i}\right|\right\}_{i=1}^{N}\right)$, where $|\cdot|$ is the Euclidean norm. This

105 procedure is illustrated in Figure 1. The diagram shows 2D contours for demonstration, but the 106 algorithm can be applied to any contiguous 3D volume. Pseudocode is shown in an additional file 107 (see Additional file 1).

108

109 The consensus CTV margin is the directional margin of the GTV to the consensus CTV. The margin 110 deviation is the coefficient of variation of the directional margins from the GTV to each expert

111 CTV. For $K$ expert CTVs and a set of directional margins $\left\{M(\boldsymbol{d})_{j}\right\}_{j=1}^{K}$ in direction $\boldsymbol{d}, \mathrm{CV}(\mathbf{d})=$ $112 \operatorname{STD}\left(\left\{M(\boldsymbol{d})_{j}\right\}_{j=1}^{K}\right) / \operatorname{MEAN}\left(\left\{M(\boldsymbol{d})_{j}\right\}_{j=1}^{K}\right)$ is the margin deviation.

\section{Application to Clinical Cases}

115 The method was applied to 11 clinical cases, chosen specifically to represent a wide range of 116 sites, and taken from a study on international practice patterns for the use of SBRT to treat non117 spine bone metastases (16). Each of nine radiation oncologists delineated the CTV contour, based 118 on the provided GTV contour and the simulation CT and MRI scans. The contours were defined 119 in the CT space (in-plane pixel size: $1.17 \times 1.17 \mathrm{~mm}$; slice thickness: $1-3 \mathrm{~mm}$ ). Using all CTV 120 contours, a consensus contour was computed using the STAPLE algorithm and approved by all 121 radiation oncologists for use in making contouring recommendations (16). 
123 For each case, directions of intraosseous and extraosseous extension were identified by two co-

124 authors ([TKN] and [CLT]), both radiation oncologists. In each direction, the consensus margin

125 was computed using the GTV and the consensus CTV, while the margin deviation was computed

126 using the GTV and the individual participant CTVs. Computations were done using MATLAB

127 (version R2016b, The Mathworks, Inc.). The median consensus margin and margin deviation for

128 intraosseous and extraosseous extension were computed across all cases.

\section{Results}

130 For illustration, Figure 2 shows the consensus margin and margin deviation in each clinician-

131 specified direction for a pubic symphysis case. This case showed no extraosseous extension.

132 Figure 3 shows the consensus margins and margin deviations over all 11 cases. The median

133 consensus margins were $7.0 \mathrm{~mm}$ (intraosseous) and $8.0 \mathrm{~mm}$ (extraosseous); the median margin

134 deviations were 0.59 (intraosseous) and 0.48 (extraosseous).

\section{Discussion}

137 The purpose of the directional margin algorithm is to quantify CTV expansions by direction for

138 the development of contouring guidelines. The advantage of applying this algorithm over

139 computing an isotropic margin is that the extent of tumor spread may depend on direction

140 because of anatomical barriers or the nature of surrounding tissue. In an application to multiple

141 cases of non-spine bone metastases the proposed method yielded separate estimates of

142 intraosseous and extraosseous extension, which would not have been possible by computing an 
143 isotropic margin. This data can inform the development of subsequent consensus contouring

144 recommendations. Since routes of spread will typically vary in different directions, anisotropic

145 CTV expansions in arbitrary directions should be a feature of future treatment planning systems.

147 The agreement between experts on the appropriate margin for a given route of spread can be 148 quantified using the margin deviation. The range of the margin deviation will need to be

149 characterized in more subjects so that outliers can be identified in future studies on contouring

150 variability that employ this technique.

152 One of the strengths of the proposed method is the ability to account for complex geometries,

153 since the lengths of all expansion vectors are incorporated (Figure 4(A)). The primary limitation

154 of this method is that it does not account for the fact that only a subset of the GTV surface is 155 relevant for margin computation when barriers to tumor spread are present in the specified 156 direction (Figure 4(B)). The method can be generalized by segmenting anatomical barriers and 157 excluding inappropriate vectors. This strategy could be facilitated by recent advances in 158 automated segmentation for CTV delineation $(15,23)$.

\section{Conclusions}

160 This report described and demonstrated a novel method to compute CTV margins and margin

161 disagreement between expert contours in any number of specified clinically relevant directions.

162 The proposed approach allows for the generation of margin recommendations in relation to 163 adjacent anatomy. The method was applied to 11 cases of non-spine bone metastases to

\section{8 of 16}


164 compute recommended intraosseous and extraosseous margins. The margin disagreement was

165 also quantified. The method can be applied in future consensus contouring studies.

\section{References}

167 1. Teh BS, Bastasch MD, Wheeler TM, Mai W-Y, Frolov A, Uhl BM, et al. IMRT for prostate 168 cancer: Defining target volume based on correlated pathologic volume of disease. Int J 169 Radiat Oncol Biol Phys. 2003;56(1):184-91.

170 2. Wang $W$, Feng $X$, Zhang $T$, Jin J, Wang S, Liu Y, et al. Prospective evaluation of microscopic 171 extension using whole-mount preparation in patients with hepatocellular carcinoma: 172 Definition of clinical target volume for radiotherapy. Radiat Oncol. 2010;5(73).

173 3. Jenkins $P$, Anjarwalla S, Gilbert $H$, Kinder R. Defining the clinical target volume for bladder $174 \quad$ cancer radiotherapy treatment planning. Int J Radiat Oncol Biol Phys. 2009;75(5):1379-84.

175 4. Soliman H, Ruschin M, Angelov L, Brown PD, Chiang VLS, Kirkpatrick JP, et al. Consensus 176 contouring guidelines for postoperative completely resected cavity stereotactic 177 radiosurgery for brain metastases. Int J Radiat Oncol Biol Phys. 2018;100(2):436-42.

178 5. Cox BW, Spratt DE, Lovelock M, Bilsky MH, Lis E, Ryu S, et al. International spine radiosurgery 179 consortium consensus guidelines for target volume definition in spinal stereotactic radiosurgery. Int J Radiat Oncol Biol Phys. 2012;83(5):e597-605. 
181 6. Redmond KJ, Robertson S, Lo SS, Soltys SG, Ryu S, McNutt T, et al. Consensus contouring 182 guidelines for postoperative stereotactic body radiation therapy for metastatic solid tumor 183 malignancies to the spine. Int J Radiat Oncol Biol Phys. 2017;97(1):64-74.

184 7. Dunne EM, Sahgal A, Lo SS, Bergman A, Kosztyla R, Dea N, et al. International consensus 185 recommendations for target volume delineation specific to sacral metastases and spinal 186 stereotactic body radiation therapy (SBRT). Radiother Oncol. 2020;145:21-9.

187 8. Lim K, Small W, Portelance L, Creutzberg C, Jürgenliemk-Schulz IM, Mundt A, et al. 188 Consensus guidelines for delineation of clinical target volume for intensity-modulated pelvic 189 190 radiotherapy for the definitive treatment of cervix cancer. Int J Radiat Oncol Biol Phys. $2011 ; 79(2): 348-55$.

9. Myerson RJ, Garofalo MC, El Naqa I, Abrams RA, Apte A, Bosch WR, et al. Elective clinical 192 target volumes for conformal therapy in anorectal cancer: A Radiation Therapy Oncology Group consensus panel contouring atlas. Int J Radiat Oncol Biol Phys. 2009;74(3):824-30.

10. Wu AJ, Bosch WR, Chang DT, Hong TS, Jabbour SK, Kleinberg LR, et al. Expert consensus 195 contouring guidelines for intensity modulated radiation therapy in esophageal and gastroesophageal junction cancer. Int J Radiat Oncol Biol Phys. 2015;92(4):911-20.

11. Leung E, D'Souza D, Bachand F, Han K, Alfieri J, Huang F, et al. MRI-based interstitial brachytherapy for vaginal tumors: A multi-institutional study on practice patterns, contouring, and consensus definitions of target volumes. Brachytherapy. 2019;18(5):598605. 
201 12. Tseng C-L, Stewart J, Whitfield G, Verhoeff JJC, Bovi J, Soliman H, et al. Glioma consensus 202 contouring recommendations from a MR-Linac International Consortium Research Group 203 and evaluation of a CT-MRI and MRI-only workflow. J Neurooncol [Internet]. 2020 Aug 29 204 [cited 2020 Aug 31]; Available from: http://link.springer.com/10.1007/s11060-020-036052056

206 13. Vinod SK, Jameson MG, Min M, Holloway LC. Uncertainties in volume delineation in 207 radiation oncology: A systematic review and recommendations for future studies. Radiother $208 \quad$ Oncol. 2016;121:169-79.

209 14. Warfield SK, Zou KH, Wells WM. Simultaneous Truth and Performance Level Estimation 210 (STAPLE): An algorithm for the validation of image segmentation. IEEE Trans Med Imaging. 211

212 15. Unkelbach J, Bortfeld T, Cardenas CE, Gregoire V, Hager W, Heijmen B, et al. The role of 213 computational methods for automating and improving clinical target volume definition. 214 Radiother Oncol. 2020 Dec;153:15-25.

215 16. Nguyen TK, Sahgal A, Dagan R, Eppinga W, Guckenberger M, Kim JH, et al. Stereotactic Body 216 Radiation Therapy for Nonspine Bone Metastases: International Practice Patterns to Guide 217 Treatment Planning. Pract Radiat Oncol. 2020;10(6):e452-60.

218 17. Allozi R, Li XA, White J, Apte A, Tai A, Michalski JM, et al. Tools for consensus analysis of 219 220 experts' contours for radiotherapy structure definitions. Radiother Oncol. 2010 Dec;97(3):572-8.

\section{1 of 16}


221 18. Rasch C, Barillot I, Remeijer P, Touw A, van Herk M, Lebesque JV. Definition of the prostate 222 in CT and MRI: a multi-observer study. Int J Radiat Oncol. 1999;43(1):57-66.

223 19. Song WY, Chiu B, Bauman GS, Lock M, Rodrigues G, Ash R, et al. Prostate contouring uncertainty in megavoltage computed tomography images acquired with a helical tomotherapy unit during image-guided radiation therapy. Int J Radiat Oncol Biol Phys.

20. Deurloo KEI, Steenbakkers RJHM, Zijp L, de Bois JA, Nowak PJCM, Rasch CRN, et al. Quantification of shape variation of prostate and seminal vesicles during external beam radiotherapy. Int J Radiat Oncol Biol Phys. 2005;61(1):228-38.

21. Jansen EPM, Nijkamp J, Gubanski M, Lind PARM, Verheij M. Interobserver variation of clinical target volume delineation in gastric cancer. Int J Radiat Oncol Biol Phys. 2010;77(4):1166-70.

22. Weiss E, Wu J, Sleeman W, Bryant J, Mitra P, Myers M, et al. Clinical Evaluation of Soft Tissue Organ Boundary Visualization on Cone-Beam Computed Tomographic Imaging. Int J Radiat Oncol Biol Phys. 2010 Nov 1;78(3):929-36.

23. Shusharina N, Söderberg J, Edmunds D, Löfman F, Shih H, Bortfeld T. Automated delineation of the clinical target volume using anatomically constrained 3D expansion of the gross tumor volume. Radiother Oncol. 2020 May;146:37-43. 
241 Figure 1 - Diagram for directional margin explanation: (A) and (B) show coronal and axial views

242 of the GTV (green) and CTV (red). In blue and orange are the expansion vectors in the anatomical

243 right $(R)$ and left $(L)$ directions respectively. The dimensions are shown along the axes. (C): The

244 vector lengths collected over the surface of the GTV. Computing the medians yields an expansion

245 of $7.0 \mathrm{~mm}$ in the $\mathrm{R}$ direction and $2.0 \mathrm{~mm}$ in the $\mathrm{L}$ direction.

246 Figure 2 - Consensus margin and margin deviation for a metastasis in the pubic symphysis: CT

247 and overlaid contours for a patient with a metastasis in the pubic symphysis. The GTV and STAPLE

248 CTV are the solid green and red contours respectively; the expert contours are dotted. The

249 relevant directions, indicated by the vectors, are termed superior-anterior (SA), inferior-posterior

250 (IP), superior-right (SR), and posterior-right (PR). The text annotations report the consensus

251 margin $(\mathrm{mm})$ followed by the margin deviation (dimensionless) associated with the same-color

252 direction.

253 Figure 3 - Comparison of intraosseous and extraosseous consensus margins and margin

254 deviations: (A) and (B) show boxplots of the consensus CTV margins and margin deviations over

255 all 11 cases of non-spine bone metastases. The points of a given color are the metrics in each

256 direction for a specific case.

257 Figure 4-Cases for discussion of strengths and limitations of method: (A): The GTV (green) and

258 STAPLE CTV (red) for an acetabular metastasis. The cyan vectors in the inferior direction show

259 variation in length, accounted for by the proposed algorithm. (B): The GTV (green) and STAPLE 
260 CTV (red) for a metastasis in the iliac crest, with the cyan vectors indicating a potential direction

261 of intraosseous extension. Some of the vectors lie outside of the bone.

263 Declarations

264 Ethics approval and consent to participate

265 This study was approved by the Sunnybrook institutional research ethics board.

266

267 Consent for publication

268 Not applicable.

269

270 Availability of data and materials

271 The datasets analyzed during the current study are available from the authors on reasonable 272 request.

273

274 Competing interests

275 CLT has received travel accommodations/expenses and honoraria for past educational seminars

276 from Elekta and belongs to the Elekta MR-Linac Research Consortium. AS has been an

277 advisor/consultant with Abbvie, Merck, Roche, Varian (Medical Advisory Group), Elekta (Gamma

278 Knife Icon), BrainLAB, and VieCure (Medical Advisor Board); ex officio Board Member to 
279 International Stereotactic Radiosurgery Society (ISRS); received honorarium for past educational

280 seminars with Elekta AB, Accuray Inc, Varian (CNS Teaching Faculty), BrainLAB, and Medtronic

281 Kyphon; research grant with Elekta AB; and travel accommodations/expenses by Elekta, Varian,

282 and BrainLAB. AS also belongs to the Elekta MR-Linac Research Consortium, Elekta Spine,

283 Oligometastases and Linac Based SRS Consortia.

285 Funding

286 This research received support from the Natural Sciences and Engineering Research Council 287 (NSERC) (RGPIN-2017-06596, CRD 507521-16). NSERC did not play a role in the design of the 288 study, nor in the collection, analysis, and interpretation of data, nor in writing the manuscript.

290 Authors' contributions

291 LSPL contributed to the formal analysis, methodology, software, and visualization. LC, RWC, and 292 AZL contributed to the methodology and supervision. AZL also contributed to funding acquisition. 293 TKN and CLT contributed to the formal analysis and methodology. AS contributed to 294 conceptualization, funding acquisition, and resources. All authors contributed to writing, 295 reviewing, and editing the manuscript.

\section{Acknowledgements}

298 Not applicable. 


\section{Additional files}

301

302 Additional file 1:

303

304 Extension: .pdf

305 Title: Directional margin algorithm pseudocode

306 Description: Pseudocode for the directional margin algorithm, which forms the basis of the 307 proposed method 
Figures

Fig. 1A

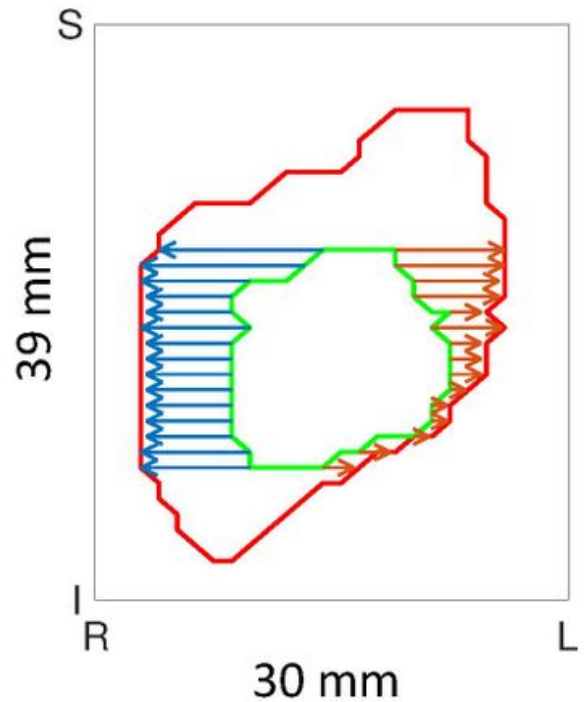

Fig. 1B

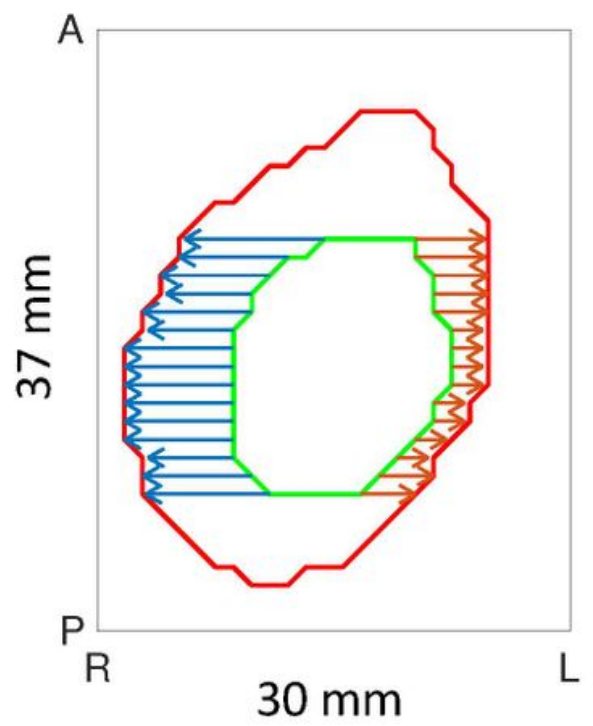

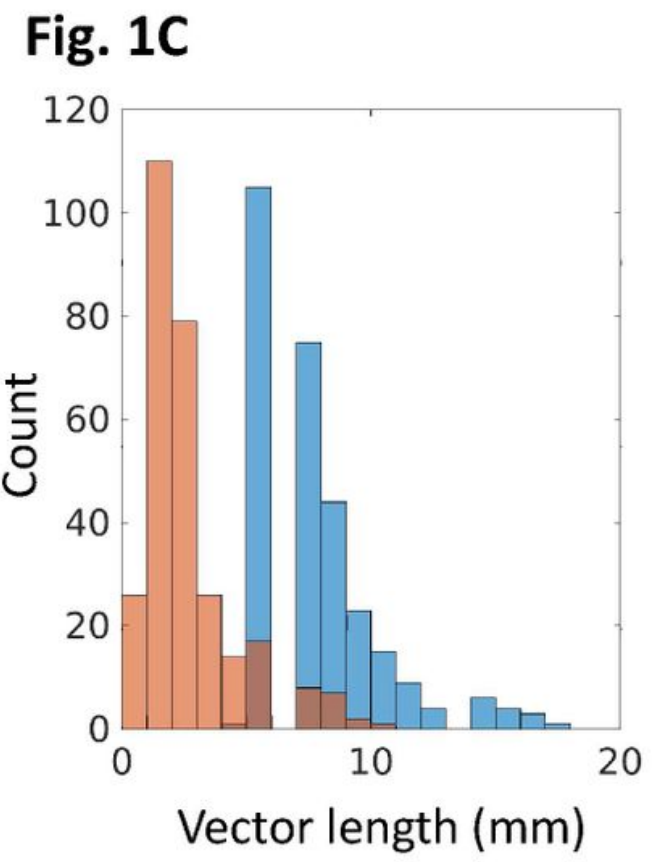

\section{Figure 1}

Diagram for directional margin explanation: (A) and (B) show coronal and axial views of the GTV (green) and CTV (red). In blue and orange are the expansion vectors in the anatomical right $(\mathrm{R})$ and left $(\mathrm{L})$ directions respectively. The dimensions are shown along the axes. (C): The vector lengths collected over the surface of the GTV. Computing the medians yields an expansion of $7.0 \mathrm{~mm}$ in the R direction and 2.0 $\mathrm{mm}$ in the $\mathrm{L}$ direction. 
Fig. 2

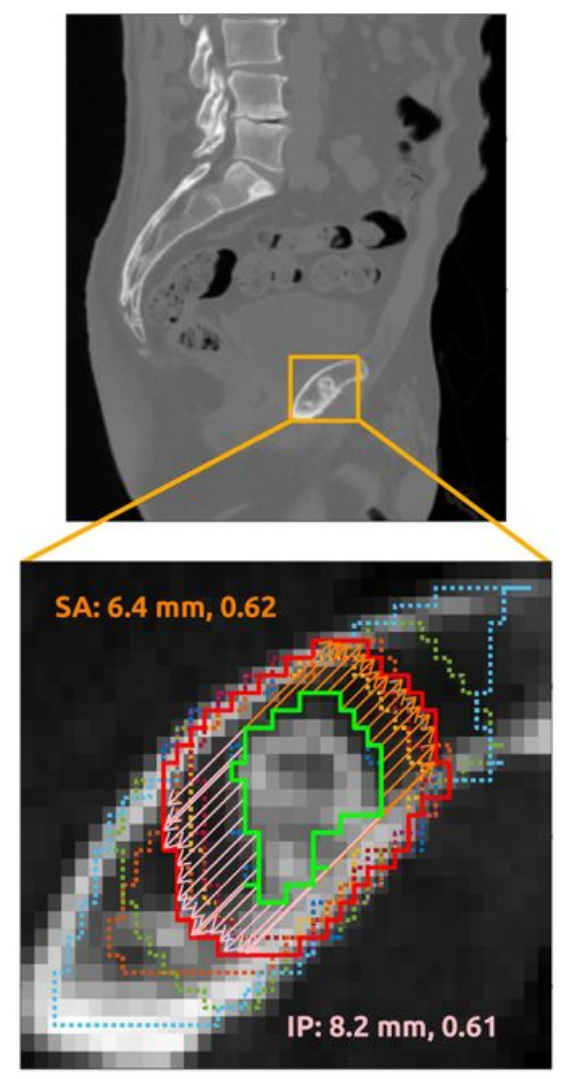

Coronal

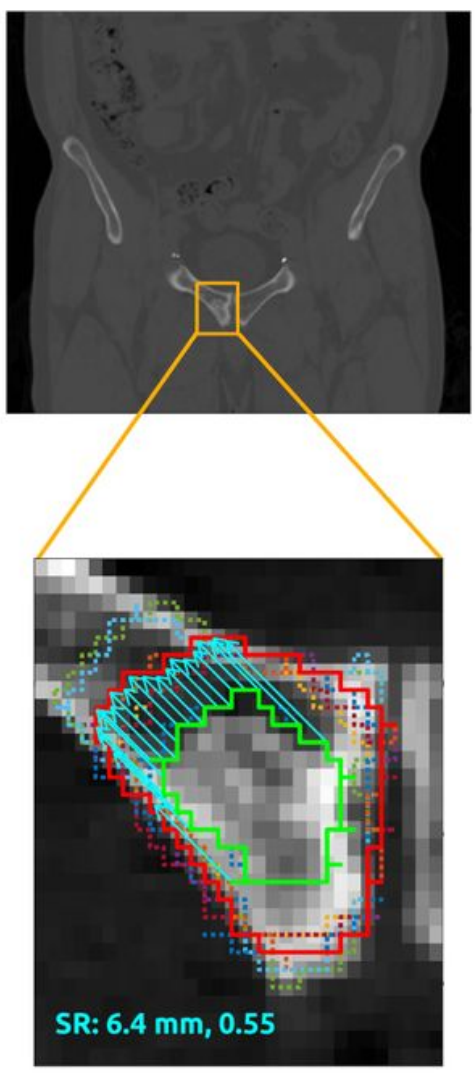

Axial

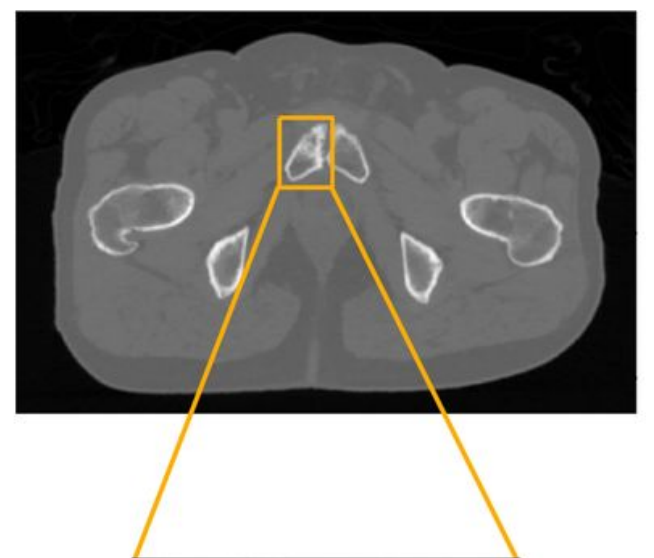

PR: $7.6 \mathrm{~mm}, 0.59$

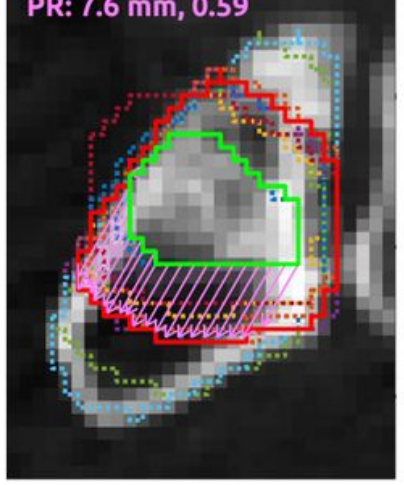

Figure 2

Consensus margin and margin deviation for a metastasis in the pubic symphysis: CT and overlaid contours for a patient with a metastasis in the pubic symphysis. The GTV and STAPLE CTV are the solid green and red contours respectively; the expert contours are dotted. The relevant directions, indicated by the vectors, are termed superior-anterior (SA), inferior-posterior (IP), superior-right (SR), and posterior-right (PR). The text annotations report the consensus margin $(\mathrm{mm})$ followed by the margin deviation (dimensionless) associated with the same-color direction. 
Fig. 3A

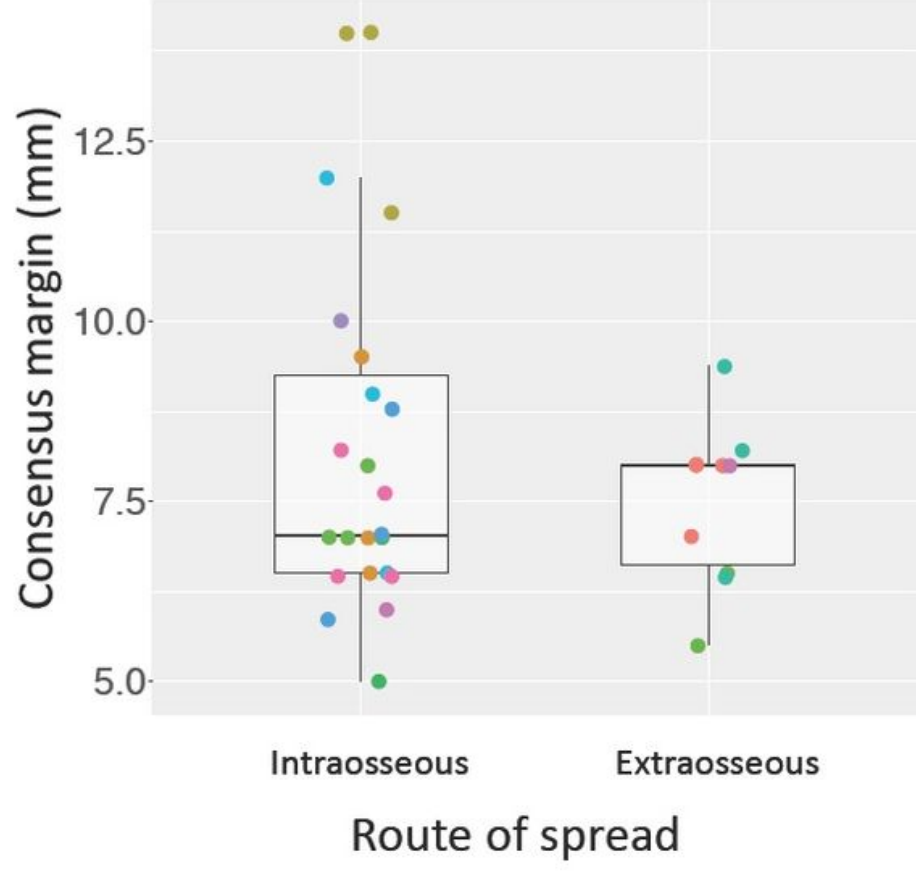

Fig. 3B

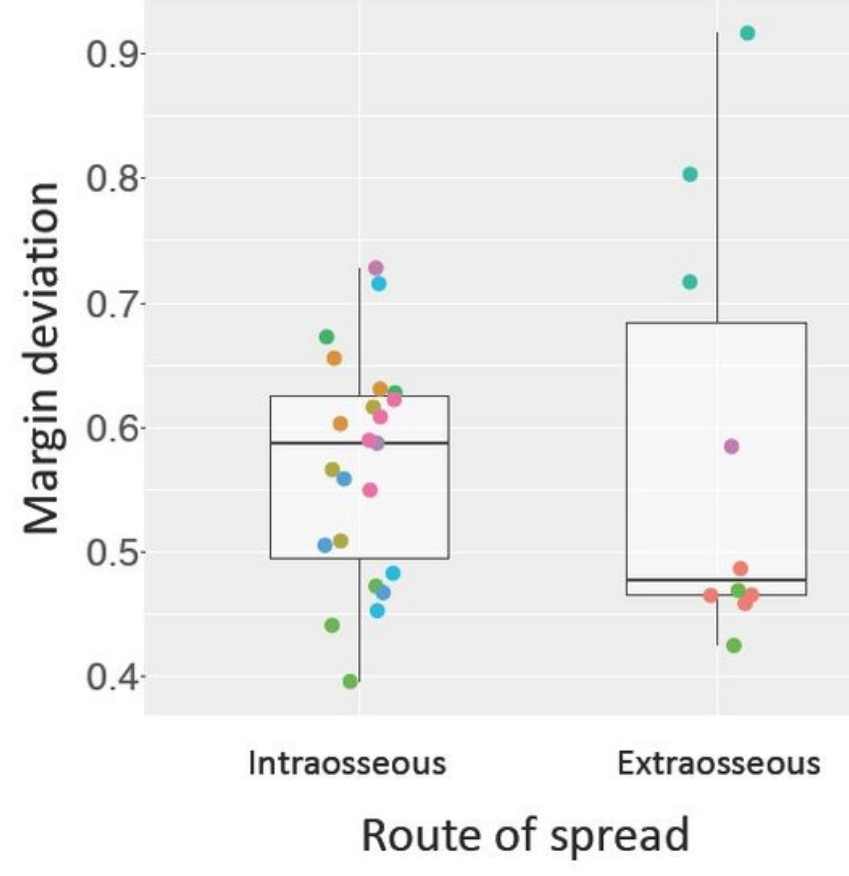

\section{Figure 3}

Comparison of intraosseous and extraosseous consensus margins and margin deviations: (A) and (B) show boxplots of the consensus CTV margins and margin deviations over all 11 cases of non-spine bone metastases. The points of a given color are the metrics in each direction for a specific case. 


\section{Fig. 4A Sagittal}

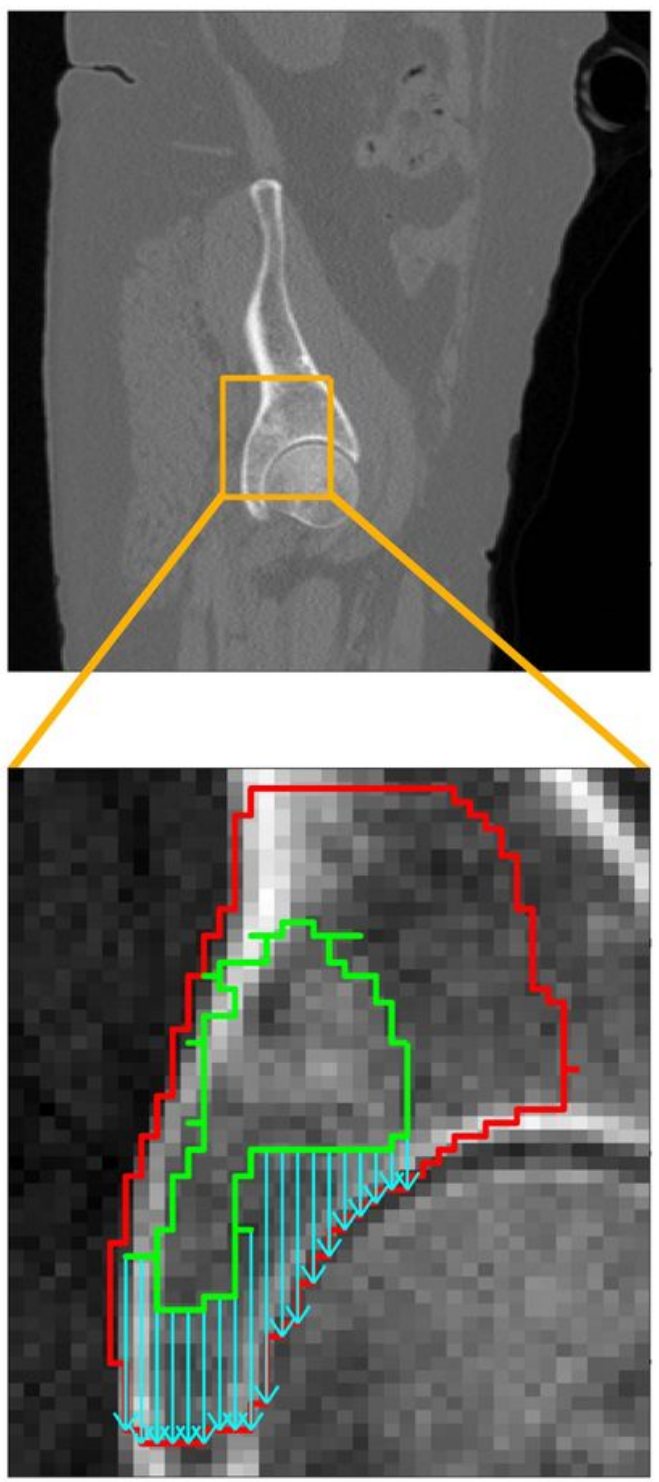

Fig. 4B

Axial

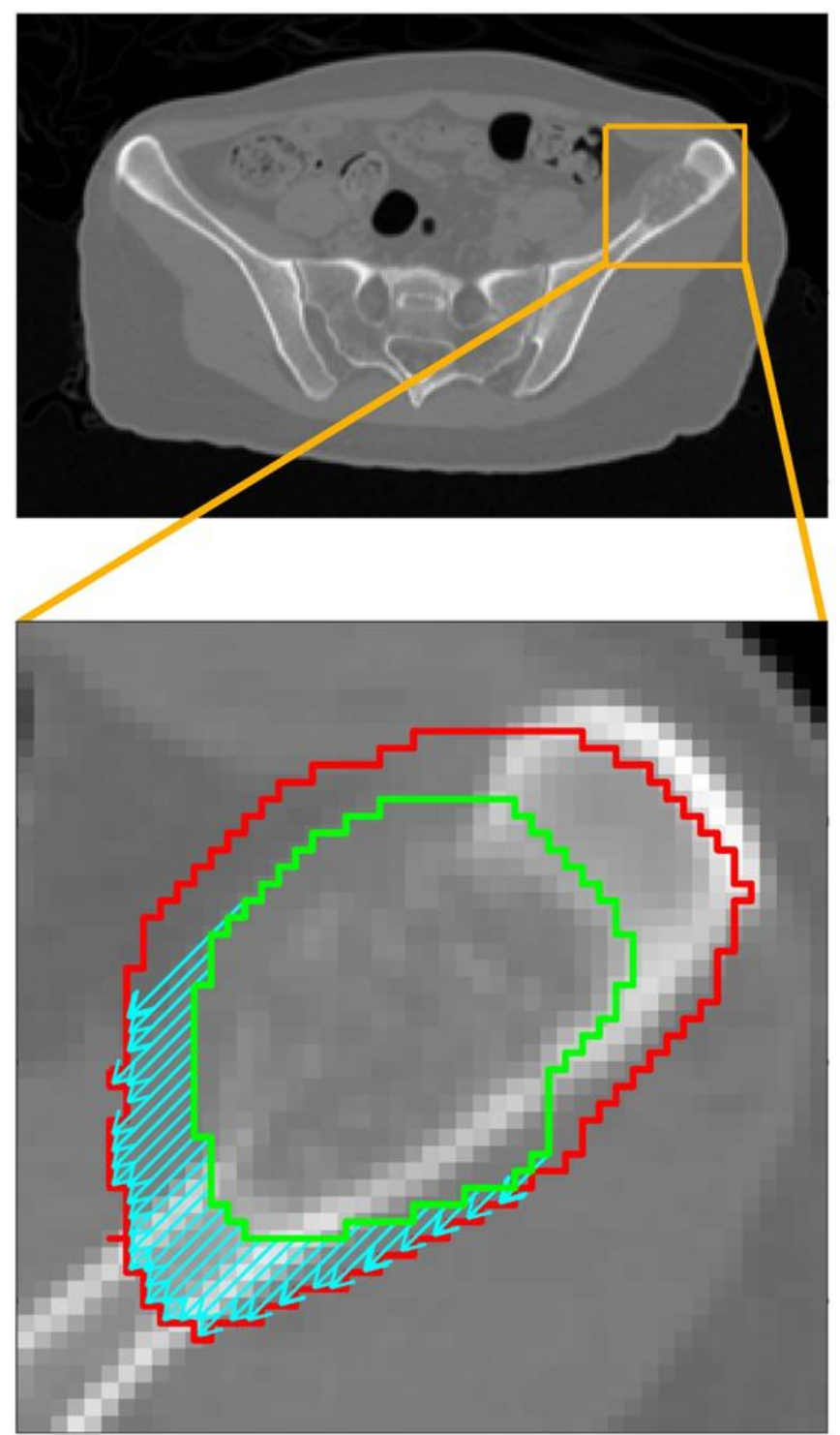

\section{Figure 4}

Cases for discussion of strengths and limitations of method: (A): The GTV (green) and STAPLE CTV (red) for an acetabular metastasis. The cyan vectors in the inferior direction show variation in length, accounted for by the proposed algorithm. (B): The GTV (green) and STAPLE CTV (red) for a metastasis in the iliac crest, with the cyan vectors indicating a potential direction of intraosseous extension. Some of the vectors lie outside of the bone.

\section{Supplementary Files}

This is a list of supplementary files associated with this preprint. Click to download.

- Additionalfile1.pdf 